Article

\title{
Synthesis of carbon nanotubes via liquid injection chemical va- pour deposition as a vector for the chemical recycling of waste composite carbon sources
}

\author{
Alvin Orbaek White ${ }^{* 1,2}$, Ali Hedayati ${ }^{1,3}$, Tim Yick ${ }^{1}$, Varun Shenoy Gangoli ${ }^{1}$, Yubiao Niu ${ }^{4}$, Sean Lethbridge ${ }^{4}$, Ioan- \\ nis Tsampanakis ${ }^{1}$, Gemma Swan', Léo Pointeaux ${ }^{1,5}$, Abigail Crane ${ }^{1}$, Rhys Charles ${ }^{5}$, Jainaba Sallah-Conteh ${ }^{1}$, An- \\ drew Anderson', Matthew Lloyd Davies, ${ }^{5,6}$, Stuart. J. Corr $7,8,9,10$ and Richard E. Palmer ${ }^{4}$
}

1 Energy Safety Research Institute, Swansea University, Bay Campus, Swansea SA1 8EN, UK; alvin.orbaekwhite@swansea.ac.uk (A.O.W.); 748963@Swansea.ac.u (T.Y.); V.S.Gangoli@Swansea.ac.uk (V.S.G); 958003@Swansea.ac.uk (I.T.); 9901886@Swansea.ac.uk (G.S.); 2124215@Swansea.ac.uk (L.P.);

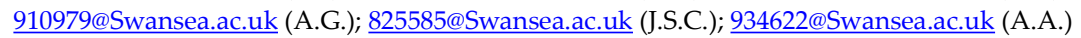

2 Chemical Engineering, Faculty of Science and Engineering, Swansea University, Bay Campus, Swansea, SA1 8EN UK; alvin.orbaekwhite@swansea.ac.uk (A.O.W.)

3 TECNALIA, Basque Research and Technology Alliance (BRTA), Alava Science and Technology Park, Leonardo da Vinci 11, 01510 Vitoria-Gasteiz, Spain; ali.hedayati@tecnalia.com (A.H.)

4 Nanomaterials Lab, Mechanical Engineering, Faculty of Science and Engineering, Swansea University, Bay Campus, Swansea, SA1 8EN, UK; R.E.Palmer@Swansea.ac.uk (R.E.P.); Yubiao.Niu@Swansea.ac.uk (Y.U.); 906934@Swansea.ac.uk (S.L.)

5 SPECIFIC, Materials Science and Engineering, Faculty of Science and Engineering, Swansea University, Bay Campus, Swansea, SA1 8EN, UK; R.Charles@Swansea.ac.uk (R.C.); M.L.Davies@Swansea.ac.uk (M.L.D.)

6 School of Chemistry and Physics, University of KwaZulu-Natal, Durban, RSA; M.L.Davies@Swansea.ac.uk (M.L.D.)

7 Department of Cardiovascular Surgery, Houston Methodist Hospital, Houston, TX, 77030, USA; sjcorr@houstonmethodist.org (S.J.C.)

8 Department of Bioengineering, Rice University, Houston, TX, 77005, USA; (S.J.C.)

9 Department of Biomedical Engineering, University of Houston, TX, 77204, USA; (S.J.C.)

10 Swansea University Medical School, Institute of Life Science 2, Swansea University, Singleton Park, Swansea, SA2 8PP UK; (S.J.C.)

* Correspondence: alvin.orbaekwhite@swansea.ac.uk

\begin{abstract}
For every three people on the planet there is approximately two Tonne (Te) of available plastic waste. We show that carbon recovery from polystyrene (PS) plastic is enhanced by the coaddition to solvents to grow carbon nanotubes (CNTs) by liquid injection chemical vapour deposition. Polystyrene was loaded up to $4 \mathrm{wt} \%$ in toluene and heated to $780^{\circ} \mathrm{C}$ in the presence of a ferrocene catalyst and a hydrogen/argon carrier gas in a 1:19 ratio. High resolution transmission electron microscopy (HRTEM), scanning electron microscopy (SEM), thermogravimetric analysis (TGA) and Raman spectroscopy were used to identify multi-walled carbon nanotubes (MWCNTs). The PS addition in the range from 0 to $4 \mathrm{wt} \%$ showed improved quality and CNT homogeneity; Raman "Graphitic/Defective" (G/D) values increased from 1.9 to 2.3; mean CNT diameters increased from 43.0 to $49.2 \mathrm{~nm}$; and maximum CNT yield increased from $11.3 \%$ to $14.2 \%$. Since both the CNT diameters and the percentage yield increased with respect to polystyrene addition, we conclude that carbon from the PS contributes to the carbon within the MWCNTs. The electrical contact resistance of acid washed Bucky papers produced from each loading, ranged from 2.2 to $4.4 \mathrm{Ohm}$, with no direct correlation to PS loading. Due to this narrow range, the materials with different loading were mixed to create six wires of an Ethernet cable and tested using iPerf to give uplink and downlink speeds of $\sim 99.5 \mathrm{Mbps}$, comparable to $\mathrm{Cu}$ wire of identical dimension ( $\sim 99.5 \mathrm{Mbps})$. The lifecycle assessment (LCA) of CNT wire production was compared to copper wire production for the use case in a Boeing 747-400 over the lifespan of the craft. Due to their lightweight nature the CNT wires decreased the $\mathrm{CO}_{2}$ footprint by $21 \mathrm{kTonne}(\mathrm{kTe})$ over the aircraft lifespan.
\end{abstract}


Keywords: carbon nanotube; plastic; chemical recycling, life cycle assessment; Ethernet; circular economy; data transmission, carbon footprint

\section{Introduction}

If carbon nantoubes (CNTs) are to be used for their lightweight and electrical conduction properties on a large/global scale [1] is there a scenario that justifies their use, especially given their (typically) large embodied energy requirement for manufacture? Moreover, in the age of sudden climatic shifts linked to carbon footprint then where would the carbon come from that is used to make the CNTs? And can the related production method from that carbon source be affective towards positive climactic outputs? To address these questions, we suggest that both plastics and solvents be used as carbon sources, and that they can create a positive environmental impact over the lifespan of their application in the aerospace sector. For instance, one of the CNTs key attributes is being lightweight with a density $1 / 6^{\text {th }}$ that of copper. As such, CNT cables are naturally lighter compared to cables made from copper, and this mass decrease will result in fuel savings for the automotive and aviation sectors when utilizing CNT wiring.

Plastic products synthesised from recycled plastics are often inferior quality compared to freshly manufactured plastics and are not feasible for the same applications. This results in fresh plastics having higher use and therefore hold greater economic value. Moreover, the recycling process is often thwarted by the inclusion fillers, pigments, and flame retardants, for example. And mixed plastics and/or composite plastic products are also challenging or not possible to recycle unless separated. To overcome this, one can consider open-loop recycling by making new products other than plastic. The most prominent technique uses thermal pyrolysis to break down the long chain polymer molecules into smaller, less complex molecules [2] via the application of intense heat [3]. This is typically carried out in the absence of oxygen to avoid the formation of undesirable carbon oxides and is done in the presence of a catalyst to increase efficiency, tailor the exact products, and improve scalability. The product mix is typically composed of oils, chars and gases that require subsequent refinement and separation, in turn necessitating further energy costs. As such, the primary focus of the field has been to improve synthesis techniques with decreased energy requirements and to create more refined and homogenous products $[4,5]$. To that end, we have developed a novel approach toward the pyrolytic growth of carbon nanotubes by including a dissolution step prior to the high temperature cycle.

CNTs growth from plastics is typically achieved using pyrolysis (Acomb 2014). Whereby, polymers such as polypropylene (PP) [6], polyethylene (PE) [7], polyethylene terephthalate (PET) [8] and high-density polyethylene (HDPE) [9] are heated in a solidgas fluidized bed reactor [10]. The resultant off-gas traverses via carrier-gas to a catalyst site to complete the conversion from vapor to solid CNT product. The pyrolytic growth of CNTs can be improved by co-addition of thermodynamically compatible solvent [1113] such as toluene $[14,15]$ to dissolve plastics including polystyrene (PS). The dissolution process confers five benefits over dry plastic pyrolysis. Polymer disentanglement initiates in the solvent [16] thus increasing reactive surface area. The polymer begins to decompose [17] and thus lowers the required energy for subsequent $\mathrm{C}-\mathrm{H}$ cleavage and consequentially allows for processing mixed plastic products. All non-soluble material crashes out of solution, such as flame retardants and other non-compatible additives, effectively cleaning the plastic prior to CNT growth. Also, once dissolved, mass transport at scale is readily achieved using pumps and pipes; therefore, the liquid injection method is beneficial for large scale operations. Moreover, mixing plastics in solvent increases carbon density which can lead to increased production capacity of CNTs via chemical recycling of mixed plastics and solvents.

In conducting a life-cycle analysis (LCA) one considers the lifespan of the material from inception to application, with particular focus on the manufacturing, transformation, use, and disposal [18]. The complexity of the phenomena involved and their interactions 
is a source of uncertainty regarding the real value of the impacts, which is why a life cycle analysis is only described as a "potential" life cycle assessment. Although considered for a long time as an experimental tool, the international standards ISO 14040 and 14044 (revised in 2006) have set the methodological and ethical bases for this type of assessment. In this age of climate uncertainty, it is imperative to adopt protocols and solutions that minimise harm compared with the problems of 'business as usual' which they are intended to solve [19].

The energy cost associated with the high entropy and exergy of plastics reconstitution is often cited as the single biggest reason to avoid plastics feedstock, especially with respect to CNT growth. Moreover, this reason is used to justify the continued use of virgin hydrocarbons due to the fact their use requires less energy by comparison; despite the fact that CNT growth from these sources never accounts for, or ever has to answer to the fact that they are derived from oil that requires extraction, purification and delivery. Though this is true in terms of energy the longer-term challenge deals with the supply of virgin materials based on oil extraction before reaching a peak oil scenario. Before that point it is prudent to establish and develop the science and technology to use pre-made plastics, in all the states in which they are found $[20,21]$; especially when they are otherwise strewn about the planet ending up in our soils and food supply [22].

Herein we report that carbon from plastics act as feedstock for carbon nanotube growth by upcycling of plastic to high-value materials via chemical process. This can be considered a viable alternative to landfill and incineration. Environmental challenges exist from both liquid and solid hydrocarbons and so we apply toluene and PS as model materials.

\section{Materials and Methods}

\subsection{The synthesis of carbon nanotubes}

Growth of multi-walled carbon nanotubes (MWCNTs) was carried out via catalytic chemical vapour deposition (CCVD) in a two-zoned horizontal liquid injection reactor (LIR), with full details described previously [23]. In summary, control CNTs were grown by injection of $1 \mathrm{~mL}(865 \mathrm{mg})$ anhydrous toluene [98\% $\left(\mathrm{C}_{6} \mathrm{H}_{5} \mathrm{CH}_{3}\right)$ Sigma Aldrich (U.K.)] at $5 \mathrm{~mL} / \mathrm{h}$ under a gas flow of $1 \mathrm{~L} / \mathrm{min}$ using blended carrier gas having $5 \mathrm{vol} \%$ hydrogen in argon [BOC U.K.] into a two-zone horizontal furnace [Nanotech Innovations SSP-354]. The first zone, used for vapour formation was set to $225^{\circ} \mathrm{C}$; the second zone used for growth was set to $780^{\circ} \mathrm{C}$. MWCNTs were grown in a $100 \mathrm{~cm}$ long quartz tube with diameter of $38 \mathrm{~mm}$ [Multi-Lab U.K.]. All reactions were carried out using needle gauge 20.

Polystyrene $\left(\mathrm{C}_{8} \mathrm{H}_{8}\right)$ n, molecular weight of 6400 [Mn 64000, Sample\#P2444-S, Polymer Source Inc. Canada] was added to toluene in concentrations of 1,2 , and $4 \mathrm{wt} \%(\mathrm{w} / \mathrm{w}) \mathrm{using}$ PS masses of $8.75,17.5$, and $35.00 \mathrm{mg}$, respectively. All reactions were carried out with a fixed catalyst ratio of ferrocene $(5 \mathrm{wt} \% \mathrm{w} / \mathrm{w})\left[98 \%\left(\mathrm{C}_{10} \mathrm{H}_{10} \mathrm{Fe}\right)\right.$ Sigma Aldrich (U.K.)] with respect to the total reactant from toluene and/or toluene and polystyrene. Prior to each growth the reactants were thoroughly mixed and degassed for 15 minutes using bath sonication. All materials were used as received without prior cracking or drying and handled as described here [24]. Each concentration of PS, and control, were grown three times to ensure trends were valid for each series. No noticeable effect or carbothermal reduction from the aging of the quartz tube was identified [25] (Figure SI1).

\subsection{The characterization and measurement of material properties}

High resolution transmission electron microscopy (HRTEM) was used to characterise as-grown samples using a Thermo Fisher Scientific Talos Transmission Electron Microscope (TEM) in high-resolution TEM mode operating at $200 \mathrm{kV}$. The TEM samples were prepared by dipping holey carbon TEM grids onto CNT powders. Fast Fourier transforms (FFT) of selected images were obtained to determine the materials' atomic structure. 
Scanning electron microscopy (SEM) was used to corroborate the presence of MWCNTs (Figure 2). A small fraction of each sample was suspended in $3 \mathrm{~mL}$ of ethanol, and $100 \mu \mathrm{L}$ of the suspension was dried on the surface of a clean silicon wafer for imaging. The SEM (JEOL 7800F FEG) was used at an operating voltage to $5 \mathrm{kV}$ or below, with a working distance of ca. $10 \mathrm{~mm}$. Diameters were measured using ImageJ [26].

A Renishaw inVia Raman microscope using a laser at $633 \mathrm{~nm}$ wavelength and 5\% beam power was used for data acquisition between $100 \mathrm{~cm}^{-1}$ and $3200 \mathrm{~cm}^{-1}$ Raman shift. The laser beam was focused by maximizing the G-peak intensity to confirm best z-height alignment of the beam between sample and detector. For each CNT sample, a Raman spectrum was acquired in three separate locations. All G/D values for that series were then averaged and reported along with the maximum G/D for that series and plotted in Figure 3. The Raman spectra also show background intensity in the region from $1400 \mathrm{~cm}^{-}$ ${ }^{1}$ to $1475 \mathrm{~cm}^{-1}$ that is due to fluorescence in amorphous carbon [27]. Line integration of the background intensity between that range was plotted to compare amorphous carbon content (Figure 3C). For each reaction condition, the individual CNT samples were probed in at least three separate locations in order to both overcome variance within a single sample and to create a significant quantity of data for comparative analysis between series.

Thermogravimetric analysis (TGA/DTA) [28] of the CNT samples was used to determine the MWCNT product yield by using ca. $10 \mathrm{mg}$ of sample placed in a platinum pan and heating under active air flow up to $800^{\circ} \mathrm{C}$. The ramp rate was $5^{\circ} \mathrm{C} / \mathrm{min}$ and hold time was $30 \mathrm{~min}$ at $765^{\circ} \mathrm{C}$. The sampling interval was set for $3 \mathrm{sec}$. MWCNT wt $\%$ is determined as the complete weight loss after full oxidation at $800^{\circ} \mathrm{C}$ in air using TGA [29] (Eqn 1).

\subsection{Preparation of CNTs for testing voltage drop}

Due to presence of residual iron catalyst witnessed in the HRTEM images an acid wash was used to remove excess iron to establish the CNT voltage drop. The oxidising acid wash, using equimolar $\mathrm{HNO}_{3}+\mathrm{H}_{2} \mathrm{SO}_{4}$ reflux $\left(70{ }^{\circ} \mathrm{C}, 24 \mathrm{hr}\right)$, strips away the amorphous carbon in addition to helping clear out the graphitic "onion" layers from the residual catalyst materials that can otherwise hinder iron from being digested [30,31]. Note that the acid wash may inadvertently damage and etch some of the MWCNTs, thus increasing the measured resistance. However, it is important to prioritise iron removal given the potential contribution towards electrical contact measurement.

\subsection{Device preparation and measurement}

Thin films were prepared using the "bucky-paper" [32] technique to measure electrical conductivity of acid washed samples. After the acid wash, the CNTs were suspended in isopropanol and CNT films were made using vacuum filtration [33]. The CNT films were dried at $80^{\circ} \mathrm{C}$ for 3 hours prior to testing and use.

Electrical resistance values were derived using Ohm's law based on measured values of voltage drop at constant current in a range of values between 0 to $100 \mathrm{~mA}$. The samples were measured along a $2 \mathrm{~cm}$ separation for all samples to ensure consistent path length.

Carbon nanotube cables were comprised of CNT powders firmly packed into the sheath of heat shrink tubing. Copper wire was inserted to the CNT wire ends and compressed to ensure maximum contact with the $\mathrm{Cu}$ lead prior to heat shrinking the outer sheath. The $\mathrm{Cu}$ leads were then used to crimp into pins and inserted into retail purchased RJ45 connectors for testing as Ethernet cable.

Quantification of the CNT Ethernet cable was conducted using iPerf3 and the cable was directly connecting two computers, both running the same Windows 10 OS update, going from a Realtek 8125B 2.5G LAN adapter capable of two-way traffic (server), to an Intel Killer E3100X LAN adapter (client); both were capable of transfer speeds up to 2500 Mbps ensuring the CNT cable could perform without being bottlenecked. A standard 10 second / 10 run test was performed to both authenticate the data transfer and measure the transfer speeds from the server (uplink) to the client (downlink). The test was repeated for statistical accuracy and the results recorded for further discussion. 


\subsection{Life Cycle Assessment(LCA) methodolgy, assumptions, and boundary conditions}

An LCA was conducted using Simapro 9.1 with Ecoinvent 3.6 as database using the method "IPCC 2013 GWP 100a (incl. $\mathrm{CO}_{2}$ uptake) V1.00". In the following LCA, the $\mathrm{CO}_{2}$ emissions (Global Warming Potential) are expressed as $\mathrm{CO}_{2}$ equivalent $\left(\mathrm{CO}_{2}\right.$ eq.) for the extraction, production, transport, use and end of life phases of CNT and copper wires. They were compared in the use case of electrical wiring in a standard Boeing 747-400 aircraft. This study includes both an analysis of the environment impact of the manufacturing of CNT wires compared to $\mathrm{Cu}$ wire and on the effect these wires have over the lifespan of a 747 in terms of $\mathrm{CO}_{2}$ generation from fuel consumption.

Assessing the potential environmental impacts of CNT manufacturing based on life cycle assessment required the following assumptions: the mass of 141 miles [34] of copper wire is $1519.6 \mathrm{~kg}$ (Supplementary information); copper ore extraction conducted in Spain; copper wire manufacturing is done in the UK within a $100 \mathrm{~km}$ radius of London. The mass of 141 miles of CNT wire is $356.2 \mathrm{~kg}$ for the same cross-section as the copper wire [35]. The raw materials for the CNTs were produced locally (within a $300 \mathrm{~km}$ radius of Swansea) and the CNTs were manufactured in the UK (using energy from the average UK electricity mix). The CNTs are produced on a laboratory scale as per the methods described herein; this is because no large scale or industrial process uses the LIR model for comparative purposes at this time, and a full study of a scaled reaction process is beyond the scope of this work.

To study the effective change in $\mathrm{CO}_{2}$ emissions from an aircraft utilising CNTs wires over $\mathrm{Cu}$ wires during its use phase required the following assumptions: an aircraft equipped with CNT wire will be 1,163.4 kg lighter. The Boeing 747-400 lifetime is approximately to be 100,000 hours of flight time with average speed of $900 \mathrm{~km} / \mathrm{h}$. Only the $\mathrm{CO}_{2}$ emissions generated during the use phase of the aircraft are considered in this analysis; accounting for the $\mathrm{CO}_{2}$ emissions during manufacture of the aircraft is beyond the scope of this work.

\section{Results and Discussion}

\subsection{Material characteristics determind using microscopy}

Using high resolution transmission electron microscopy, we observe the presence of both carbon nanotubes and residual metal catalyst particle (Figure 1). The number of walls ranged between 18 and 52, with CNT diameters ranging from 18 to $45 \mathrm{~nm}$, there was no apparent trend associated with PS loading (within the small number of TEM images counted). Several MWCNTs displayed closed caps, and some had several catalyst particles within a single CNT (Figure 1B).

The longest single CNT observed was $13.7 \mu \mathrm{m}$ (Figure 1E), although longer CNTs may exist. The $0.34 \mathrm{~nm}$ spot in the FFT is due to the $\mathrm{d}(002)$ lattice spacing associated with multiple graphitic walls, this is significant because it further verifies the presence of nanotubes based on their crystallography. And the $0.17 \mathrm{~nm}$ spot is the $\mathrm{d}(004)$ spacing from the second order diffraction of the $\mathrm{d}(002)$ lattice. However, it is more challenging to differentiate iron oxide compounds such as $\mathrm{Fe}_{2} \mathrm{O}_{3}, \mathrm{Fe}_{3} \mathrm{O}_{4}$ and $\mathrm{FeOOH}$ from FFTs as they have very similar spots/d-spacings (Figure SI2-SI4).

Using scanning electron microscopy (SEM), one can observe the materials easily identified as CNTs based on their tangled nature and long morphology (Figure 2). The catalyst content could also be identified due to higher charging density around the Fe and $\mathrm{FeC}$ structures. Some of the catalyst could be seen within the CNTs structures, further confirming nanotube formation due to the presence of hollow cores. In some cases, the catalyst could be seen as high intensity bright spots both along the inside walls of the 

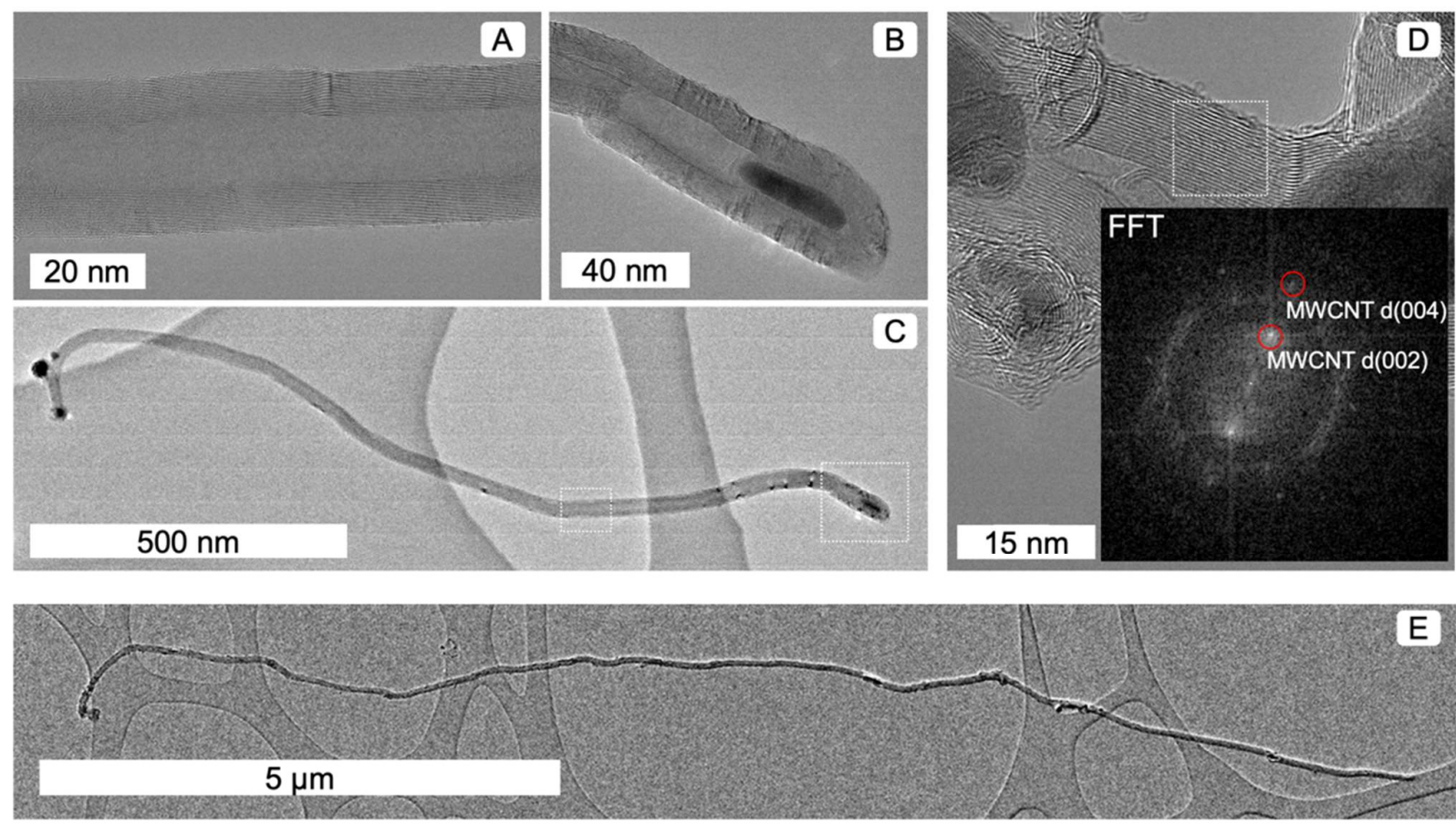

Figure 1. High resolution Transmission Electron Microscope images showing A) multiple walls and B) having the presence of internal catalyst particles in C) long MWCNTs. D) FFT analysis confirmed d(002) and d(004) line spacings. MWCNT lengths E) reach over $10 \mu \mathrm{m}$ in this case.

CNTs and at the ends. Most of the CNTs displayed a relatively tortuous path once deposited on the SEM stubs for imaging. In the control samples, the average diameters was found to be $43.0 \mathrm{~nm}+/-15.2 \mathrm{~nm}$ (Std. Dev). Interestingly, at low PS (1 wt\%) concentration the CNT diameters decreased with a concurrent smaller distribution resulting in MWCNTs with $39.4 \mathrm{~nm}+/-14.5 \mathrm{~nm}$ average diameter. However, increased PS content led to increased diameters and greater standard deviations. The increase in diameter can be accounted for by the increase in carbon concentration in the feedstock.
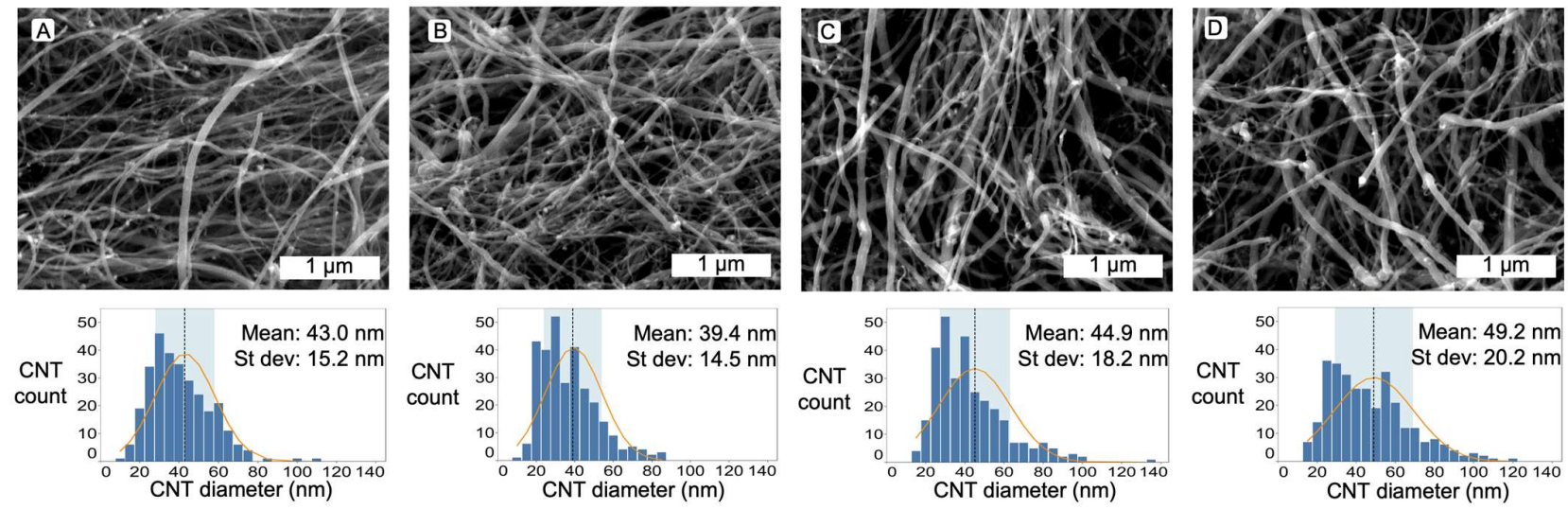

Figure 2. Representative scanning electron microscope images of carbon nanotubes made from A) control with zero PS, B) PS $1 w t \%, C)$ PS $2 w t \%$ and D) PS $4 w t \%$. Along with histogram data for each sample showing mean and standard deviation for each sample.

\subsection{Material characteristics determined using spectroscopy}

Raman spectroscopy is commonly used to measure and characterise the fingerprint peaks associated with MWCNTs (Figure 3A). It can also be used to make comparative analysis of amorphous carbon content (Figure 3B) between samples. More importantly, 
resonant Raman spectroscopy is used to quantify the quality of the MWCNTs by direct comparison of G- and D-peak intensities (Figure 3C).

The representative spectra for all four conditions all exhibit G- and D-peaks associated with the presence of MWCNTs (Figure 3A). In the case of MWCNTs, the G-peak (also referred to as $\mathrm{G}$ mode, or Graphitic mode) appears in the $1500-1600 \mathrm{~cm}^{-1}$ range due to the tangential displacement of $\mathrm{C}-\mathrm{C}$ bond stretching, effectively indicating the density of $\mathrm{sp}^{2}$ hybridized carbons atoms that exist in the CNT lattice, and along the circumferential direction of the nanotube. However, the D-peak, or Disorder mode, observed at 1290-1330 $\mathrm{cm}^{-1}$ represents conversion of carbon centres from $\mathrm{sp}^{2}$ to $\mathrm{sp}^{3}$ hybridisation states that can occur due to a break in the symmetry of the graphite plane [36]. Therefore, comparing the peak heights of the G- and D-peak effectively quantifies the graphitic versus the defective carbon atoms within the CNT lattice. The D peaks intensities must be taken with respect to the $\mathrm{G}$ peak intensities. When the $\mathrm{D}$ peaks are high it indicates a large portion of $\mathrm{sp}^{3}$ carbon atoms. $\mathrm{sp}^{3}$ carbon atoms can arise for several reasons relating to the broken symmetry of the graphitic structure. For this reason, the D-peak is used to refer to the defects in the CNT structure. It is not that the CNTs are themselves defective, but that they have imperfections within their body. A third fingerprint peak is also evident in Figure $3 \mathrm{~A}$, and this $G^{\prime}$ (G prime) peak corresponds to disorder induced carbon features arising from finite
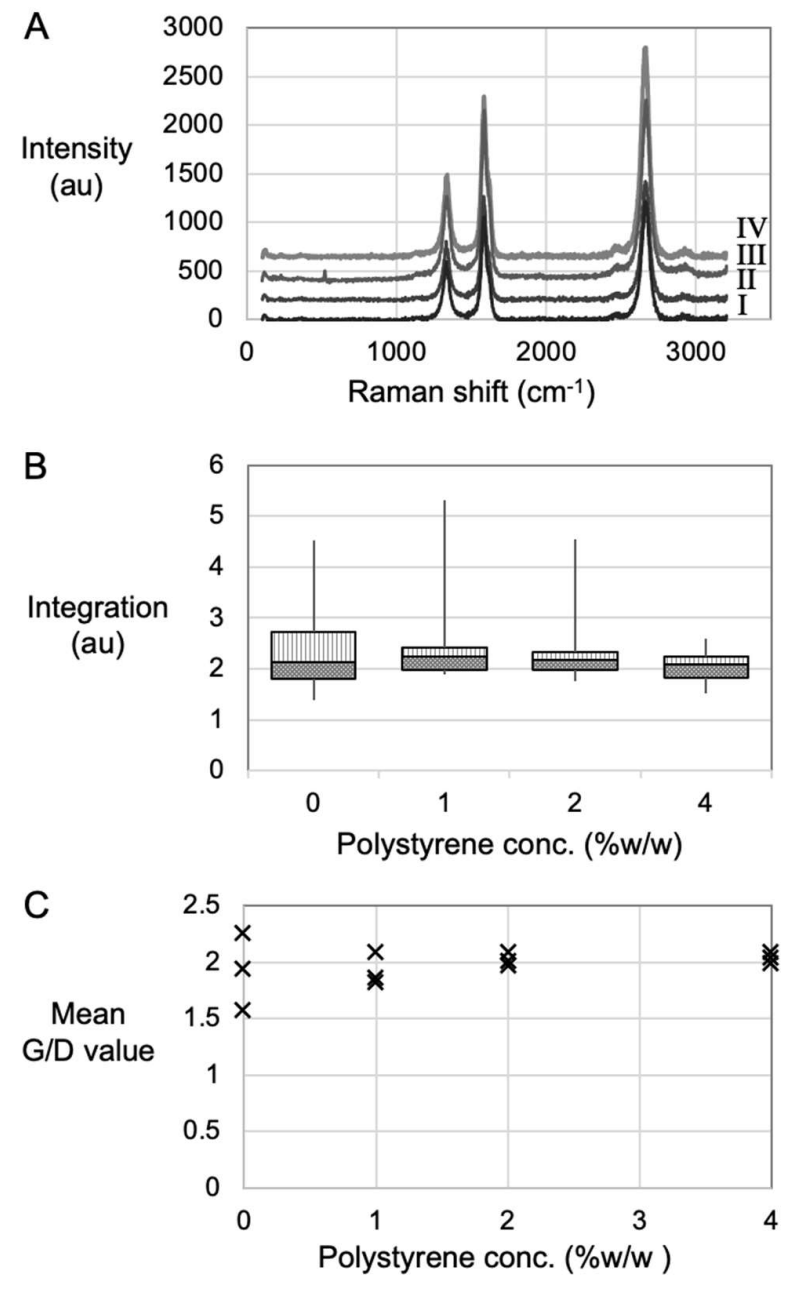
particle size distribution or lattice distortions in the CNTs. Moreover, the presence of G' peak further indicates the presence of multiple walls which is to be expected for samples of multi-walled carbon nanotubes.

The spectral background intensity in the range $1400-1475 \mathrm{~cm}^{-1}$ occurs due to fluorescence of amorphous carbon and following line integration is used to compare amorphous carbon concentration between samples [37]. A Box Plot [38] (Figure 3B) of the line integration values indicates smaller and narrowing range with higher PS loading. As small integration value indicates less amorphous carbon content, we conclude that amorphous carbon decreases as a consequence of increased PS loading.

The G/D value indicates quality in bulk samples since a large G-peak relative to the D-peak indicates a strong resonance condition of $\mathrm{sp}^{2}$, graphitic carbon. [39] Comparisons between reaction conditions can be made in terms of range variance and minimum versus maximum values for each reaction condition. The mean G/D values for the control group suggest on average this condition renders highest quality materials, but they range from 1.5 to 2.3, suggesting they are heterogeneous samples compared to the PS $4 \mathrm{wt} \%$ condition that have an average range from 1.9 to 2.1. Moreover, PS samples generally display a tightening of data points which suggests greater homogeneity. The G/D values improved with a tightened range too, indicating higher quality materials were created by the use of PS whereby the maximum G/D value was recorded using the PS $4 \mathrm{wt} \%$ (Table 1). Moreover, the increase of PS concentration may not be detrimental, based on the steady measurement of the mean value at 2.0, even after the increase.

Figure 3. Raman data showing A) typical spectra stacked from I) Control, II) PS 1wt \%, III) PS 2 wt $\%$ and IV) PS 4 wt $\%$. B) Box plot from line integration between 1400-1475 $\mathrm{cm}^{-1}$, and C) Mean G/D values. All spectra acquired using $633 \mathrm{~nm}$ laser. 
Table 1. This is a table. Tables should be placed in the main text near to the first time they are cited.

\begin{tabular}{lrrrr}
\hline & \multicolumn{4}{c}{ PS concentration (wt\%) } \\
& 0 & 1 & 2 & 4 \\
\cline { 2 - 5 } Mean G/D value & 1.9 & 2.0 & 2.0 & 2.0 \\
Maximum G/D value & 2.3 & 2.3 & 2.3 & 2.5 \\
\hline
\end{tabular}

\subsection{Material properties determined using mass balance and thermogravimetric analysis}

The solid product was weighed using mass balance and used to determine MWCT percentage yield according to Equation 1. TGA data based on the oxidization, and subsequent mass loss at temperatures between $400-600^{\circ} \mathrm{C}$ is characteristic of MWCNTs (Figure 4). Residual mass in the TGA pan is the oxidised catalyst material, typically orange in colour, it is represented by a residual mass percentage which used to calculate MWCNT percentage yield (Equation 2). For example, using $4 \mathrm{wt} \%$ of PS, $130 \mathrm{mg}$ was recovered from the reactor. A TGA test shows $6.53 \%$ to be residual catalyst, this equates to a MWCNT mass of $121.55 \mathrm{mg}$, which in turn corresponds to a CNT percentage yield of $14.2 \%$ (Equation 2). All the values are tabulated and averaged to determine the average CNT product yield (Table 2). Since we are dealing with catalytic processes where some

Thermogravimetric data of CNT-PS-4wt\%

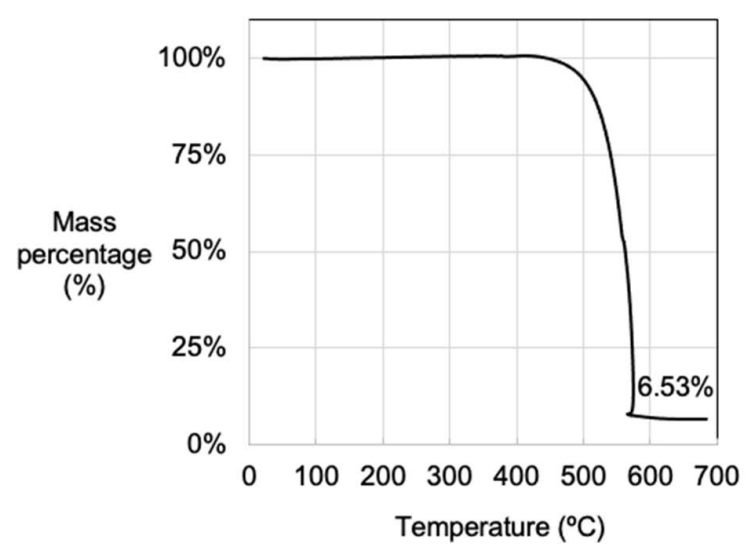

reactions have outstanding results compared to the norm/average, we have also reported the maximum percentage yields. Though not the norm, they represent the best-case scenario. Based on TEM observations, we assume negligible quantities of amorphous carbon were present and this assumption is reinforced by the line integration study in the Raman spectra.

Based on the aforementioned observations that increased PS loading, and therefore higher carbon density samples, leads to MWCNT with wider walls (Figure 2), with lower amorphous carbon content (Figure 3B) and higher quality due to higher maximum G/D values (Figure $3 C$ ); that carbon from the PS becomes carbon in the MWCNTs. This correlates with the both the Baker model [40] of carbon fiber growth and the Puretzky model [41] of CNT growth whereby as more carbon enters the reaction, more walls are created.

Figure 4. Thermogravimetric data of CNT growth from polystyrene $4 \mathrm{wt} \%$ loading, residual mass found to be $6.53 \%$, composed of iron oxide residue from catalyst precursor.

$$
\begin{aligned}
& \text { CNT percentage yield }=\frac{\left(\text { Mass }_{\text {Product }}\right)-\left(\text { Mass }_{\text {Fe residue }}\right)}{\text { Mass }_{\text {Theoretical yield }}} \times 100 \\
& \text { CNT percentage yield }=\frac{130(\mathrm{mg})-8.45(\mathrm{mg})}{856.71(\mathrm{mg})} \times 100=14.2 \%
\end{aligned}
$$

Table 2. Mass data and percentage yield results for CNTs grown using polystyrene.

\begin{tabular}{lrrrr}
\hline Sample & Mass of carbon reactant (mg) & Average CNT product (mg) & Maximum CNT product (mg) & Maximum CNT yield (\%) \\
\hline Control & 823.05 & 89.80 & 92.80 & $11.3 \%$ \\
$1 \mathrm{wt} \%$ PS & 831.47 & 80.47 & 89.36 & $10.7 \%$ \\
$2 \mathrm{wt} \%$ PS & 839.88 & 100.36 & 107.83 & $12.8 \%$ \\
$4 \mathrm{wt} \%$ PS & 856.71 & 106.58 & 121.55 & $14.2 \%$ \\
\hline
\end{tabular}




\subsection{General mechanism of carbon nanotube growth}

The CNT growth mechanism occurs via a multi-step process involving the formation of catalysts, the decomposition of carbon sources and the reconstitution of carbon to a nanotube structure. This process begins with the in-situ decomposition of ferrocene to render a mixture of $\mathrm{Fe}_{2} \mathrm{O}_{3}$ and $\mathrm{Fe}_{3} \mathrm{O}_{4}$ catalytic particles. The iron particles agglomerates and form different sized structures ranging from nanoparticles to micro particles. These iron particles act as catalytic surfaces known to catalytically cleave the $\mathrm{C}-\mathrm{H}$ bonds in hydrocarbon reactants such as plastics, polymers and toluene. The lifetime and activity of the catalyst is maintained by using a constant flow of hydrogen in the gas stream that both simultaneously reduces the metal, making it active for carbon cracking, and attracts errant carbon moieties that might otherwise saturate the catalyst, leading to coking and eventual catalyst poisoning. Complete control over the $\mathrm{CNT}$ products is highly challenging because catalysts highly structure-sensitive [42] materials, even slight variance from the optimum condition can lead to drastic changes in product. A key struggle with CNT growth is the inverse relationship between sample yield and sample quality - it is consistently one or the other.

Mechanistic understanding of carbon nanotube growth suggests that carbon in the form of $\mathrm{C} 2[43,44]$ enters into the catalyst lattice and saturates the molten metal crystal. Following saturation within the catalyst, carbon precipitation forms at a growth facet, typically a high energy facet such as $\langle 111>$; exiting the catalyst in graphitic tubular form. The tubular morphology of the carbon is physically bounded by the outer edges and outer diameter of the catalyst particles and the graphitic form is adopted because of the low entropy state of graphite.

\subsection{Contribution to CNT growth from Toluene}

Toluene decomposition compliments CNT growth from plastics in two distinct ways. Firstly, in the liquid phase it decomposes the polymer to monomers, and in the gas phase it readily forms $\mathrm{C} 2$ units leading to $\mathrm{CNT}$ nucleation. CNT nucleation is notably a function of hydrocarbon thermal decomposition to suitable fragments (C2 units). C2 formation from thermal decomposition of toluene [45-47] can occur via a number of pathways. For example, toluene can decompose either through benzyl radical (Equation. 3) or phenyl radical (Equation. 4) formation; however, the former dominates at the temperatures studied herein. The benzyl radical undergoes subsequent decomposition to form C5, C3, and $\mathrm{C} 4$ units, that can in turn be decomposed further, Equation. 5 and 6. Toluene decomposition leads to formation of $\mathrm{C} 2, \mathrm{C} 3$ and $\mathrm{C} 4$ units that will decompose either in the gas phase due to temperature or via radical attack, or on the catalyst particle surface, to render the C2 fragments associated with nanotube growth. Moreover, the highly active C2 units or radicals mentioned herein can initiate and advance PS decomposition too.

$$
\begin{aligned}
& \mathrm{C}_{6} \mathrm{H}_{5} \mathrm{CH}_{3} \rightarrow \mathrm{C}_{6} \mathrm{H}_{5} \mathrm{CH}_{2}{ }^{*}+\mathrm{H}^{*} \\
& \mathrm{C}_{6} \mathrm{H}_{5} \mathrm{CH}_{3} \rightarrow \mathrm{C}_{6} \mathrm{H}_{5}{ }^{*}+\mathrm{CH}_{3}{ }^{*} \\
& \mathrm{C}_{6} \mathrm{H}_{5} \mathrm{CH}_{2}{ }^{*} \rightarrow \mathrm{C}_{5} \mathrm{H}_{5}+\mathrm{C}_{2} \mathrm{H}_{2} \\
& \mathrm{C}_{6} \mathrm{H}_{5} \mathrm{CH}_{2}{ }^{*} \rightarrow \mathrm{C}_{3} \mathrm{H}_{3}+\mathrm{C}_{4} \mathrm{H}_{4}
\end{aligned}
$$

\subsection{Contribution to CNT growth from Polystyrene}

PS is a conjugated aromatic polymer structure that can be decomposed by scissioning the conjugated chain into the styrene monomer units via $\mathrm{H}^{*}$ attack [48]. $\mathrm{H}^{*}$ originates from several sources both in the gas and liquid phase. For example, liquid phase decomposition of toluene (Equation 3) liberates $\mathrm{H}^{*}$, especially during the sonication used in the mixing of the liquid or at elevated temperatures. The temperature in the CVD furnace vaporizes toluene, resulting in further $\mathrm{H}^{*}$ release which compliments $\mathrm{H}^{*}$ originating from the thermal splitting of hydrogen in the gas flow. Further $\mathrm{H}^{*}$ can be formed at elevated 
temperatures by catalytic cracking of hydrocarbon over iron oxide catalyst [49]. Even without catalyst presence, thermal cracking of the polymer produces an aromatic product state consisting of high concentrations of styrene $(\sim 50-79 \mathrm{wt} \%)$ together with the styrene dimer and trimer and other aromatic compounds including toluene, xylene and alkylated benzenes [50]. There is a complimentary cascade of chemical reactions induced by various pathways to create $\mathrm{H}^{*}$ that assist in the decomposition of polystyrene to styrene monomers. Once decomposed to styrene monomer or similar hydrocarbon they are further decomposed to C2 units in order for CNT growth to occur.

\subsection{Carbon nanotube devices measurement and application}

In all cases, the voltage increased linearly with current indicating ohmic resistance of the CNT films. As a reference, the electrical resistance of a retail piece of copper tape was measured by the same procedure. The electrical resistance of the CNT samples were found to be in the range of 2.4 to $4.4 \Omega$. This is two orders of magnitude higher than the copper reference sample. On average the use of PS increased electrical resistance (Figure SEI1). However, the best conductivity performance came from sample PS-2 (2 wt $\% \mathrm{w} / \mathrm{w})$ of 2.4 $\Omega$ compared to the control $R=0.6 \Omega$. Although these values are generally higher than that of copper, the mass difference between carbon and copper make these materials an attractive alternative to copper once the $I^{2} R$ losses are improved, especially since their lightweight nature is critical. Moreover, it is likely our attempt to remove contributions from catalyst particles [51] (used as catalyst) by virtue of the acid wash may inadvertently have led to the poor electrical properties. The oxidizing acid wash may have created additional sidewall defects that ultimately hinder electron transport. This may be why no apparent trend in electrical performance is noted wrt to PS loading in the feedstock.

By quantifying the uplink and downlink speed, using iPerf, we conclude that MWCNT wires are capable of data transfer rates of at least $99 \mathrm{Mbps}$. Based on our findings, the $\mathrm{Cu}$ wire gauge used as well as the RJ45 connectors are holding back the potential of the CNT wires themselves as determined by the fact $\mathrm{Cu}$ wires, once connected to identical RJ45 connectors, also maxed out at $94.7 \mathrm{Mbps}$ (Table 3). Industrial grade RJ45 connectors certified for the CAT7 standards coupled with higher quality, thicker gauge copper wiring as well as better assembly and crimping will help negate the bottleneck. As it stands, the CNT Ethernet cables can adhere to the rated uplink and downlink speeds of the CAT5 standard, with results saturating at the 100 Mbps value (Figure SI5, Video SI1) which has been seen previously [52]. These are speeds are adequate for UK adoption and Broadband classification as determined by the UK Government regulator [53].

Table 3. Ethernet speed tests using three devices, one (Cat6) commercial device and two lab made devices using CNTs or CU wire as active transmission component.

\begin{tabular}{lrr}
\hline & Maximum uplink speed (Mbps) & Maximum downlink speed (Mbps) \\
\cline { 2 - 3 } Cat6 & 998 & 995 \\
CNT cable & 94.9 & 97 \\
Cu cable & 92.7 & 94.7 \\
\hline
\end{tabular}

\subsection{The life cycle assessment of MWCNT growth and wire production versus $C u$ wire formation}

What is the impact of making CNT wires (lab scale) compared to making equivalent length of $\mathrm{Cu}$ wire from an industrial process? Moreover, what carbon saving could be achieved on account of the lighter aircraft over its typical lifespan and where is the breakeven point for using CNT wires instead of $\mathrm{Cu}$ wire? For every $1 \mathrm{~kg}$ of CNT powder, 1.58 $\mathrm{kg}$ of $\mathrm{CO}_{2}$ is created (Supplementary information) which is largely due to the electricity requirement for heating $[54,55]$. The electricity sources available in the UK did not make a significant impact on decreasing the $\mathrm{CO}_{2}$ generation (Supplementary information). Using the CNT powder to make 141 miles of cabling, $356.2 \mathrm{~kg}$ of $\mathrm{CNTs}$, resulted in $\mathrm{CO}_{2}$ 
emissions of 545 metric Tonne (Te) compared to making 1,519.6 kg of copper wire which generated 12 Te of $\mathrm{CO}_{2}$; this is nearly 45 times more $\mathrm{CO}_{2}$ just from the manufacturing of $\mathrm{CNT}$ cables compared to $\mathrm{Cu}$ wires (Figure 5A). However, once economies of scale such as Wright's Law [56,57] are considered we anticipate CNT cable manufacturing at larger scale to decrease $\mathrm{CO}_{2}$ emissions further [58].

Other environmental factors aside from $\mathrm{CO}_{2}$ emission exist such as (but not limited to): freshwater eutrophication, freshwater ecotoxicity, marine ecotoxicity and stratospheric ozone depletion. When the normalised sum [59] of these impacts is compared between CNT wires (at lab scale) and $\mathrm{Cu}$ wires one can note an approximate eight-fold increase in negative impacts from $\mathrm{Cu}$ wire manufacture (Figure 5B).

\subsection{The life cycle assessment of use case of MWCNTs in a Boeing 747-400 aircraft}

Using the CNT wires make the Boeing 747-400 series 6\% lighter in mass, and this mass saving improves fuel efficiencies such that after just 2,585 hours the $\mathrm{CO}_{2}$ emission decreases when using CNT wires (Figure 5C). This savings could be improved once the wire manufacturing process were scaled by virtue of scaling laws in manufacturing proficiencies. Note that almost $99 \%$ of the $\mathrm{CO}_{2}$ eq. emissions are $\mathrm{CO}_{2}$ emissions (of the $4520 \mathrm{kTe}$ of $\mathrm{CO}_{2}$ eq. emitted by the plane, $4450 \mathrm{kTe}$ are $\mathrm{CO}_{2}$ emissions). Due to the lightweight nature of CNT wires compared to heavier $\mathrm{Cu}$ wires, over the life span of the aircraft $(100,000 \mathrm{hr})$, the use of CNT wires decreases the carbon footprint by $21 \mathrm{kTe}$ less $\mathrm{CO}_{2}$.

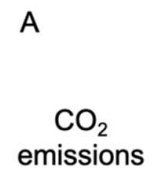

(Te)

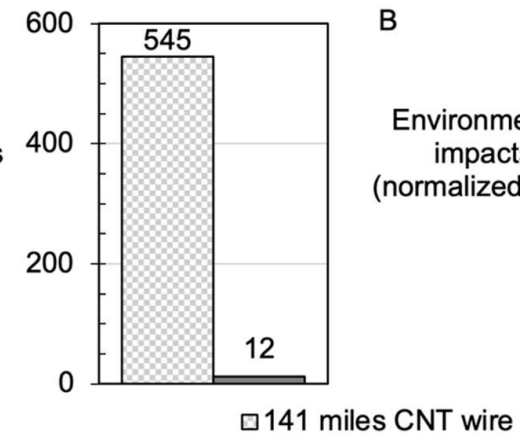

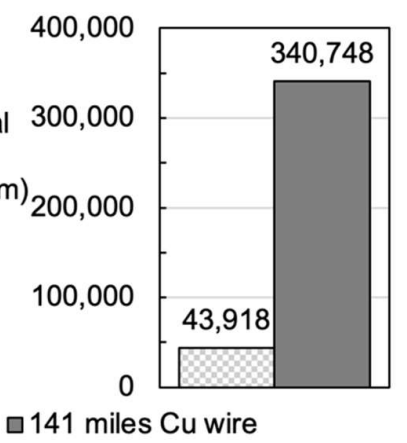

\begin{tabular}{rrr} 
C & \multicolumn{3}{c}{} \\
$\mathrm{CO}_{2}$ emissions of 747 aircraft $(\mathrm{kTe})$ \\
Time (hr) & Cu wire & CNT wire \\
\hline 0 & 0.012 & 0.545 \\
100 & 4.529 & 5.087 \\
500 & 22.591 & 23.060 \\
1,000 & 45.170 & 45.526 \\
2,000 & 90.327 & 90.459 \\
2,585 & 116.744 & 116.744 \\
20,000 & 903.155 & 899.242 \\
100,000 & $4,515.723$ & $4,493.833$
\end{tabular}

Figure 5. Life cycle analysis comparing A) $\mathrm{CO}_{2}$ emissions and (B) normalized sum of all environmental impacts between the production of 141 miles of CNTs wires versus $\mathrm{Cu}$ wire. Comparative table $\mathrm{C}$ ) showing the $\mathrm{CO}_{2}$ emission (kTe) per copper versus $\mathrm{CNT}$ wires.

\section{Conclusions}

Multi-walled carbon nanotubes were grown using liquid injection chemical vapour deposition at $780{ }^{\circ} \mathrm{C}$ using ferrocene catalyst particles to convert toluene and various PS concentrations. Greater (PS) concentrations increased MWCNT diameters, strongly suggesting carbon from the plastic incorporates into the MWCNTs. The maximum Raman G/D values both increased and the range of mean values narrowed which suggests higher quality materials with greater homogeneity were created with respect to higher PS concentration; this is likely due to the highly reactive nature of styrene monomers. This synthesis method improved MWCNT quantity without incurring a measurable loss in quality. MWCNT Ethernet cables were found to have $99.5 \mathrm{Mbps}$ uplink and downlink speeds, comparable to $\mathrm{Cu}$ wires of similar diameter. A life cycle assessment (LCA) of the MWCNT wires made from PS suggests electricity to power the CVD furnace is the largest embodied impact. Moreover, the LCA determined that a $21 \mathrm{kTe} \mathrm{CO}_{2}$ footprint could be saved over the lifespan of a Boeing 747-400 due to the aircraft have reduced weight by using CNT wires. 


\section{Patents}

Two patents have been filed from this work. A.H. and A.O.W. filed: PROCESS FOR REUSE OF PLASTIC THROUGH THE CONVERSION TO CARBON NANOMATERIALS United States Patent Application 20190375639; T.Y. and A.O.W filed: CABLES AND METHODS THEREOF United States Patent Application 20210158995. Both have been filed for PCT status.

Supplementary Materials: The following are available online at www.mdpi.com/xxx/s1, Figure SI1: MWCNT yield versus order of the reaction, Figure SI2: FFT of HRTEM images, Figure SI3: FFT of HRTEM images, Figure SI4: FFT of whole HRTEM image, Figure SI5: Voltage drop date for CNT films, Figure SI5: Picture of the CNT Ethernet Cable; Discussion on potential environmental impacts of CNT manufacturing, Discussion on Fossil $\mathrm{CO}_{2}$ eq and $\mathrm{CO}_{2}$ uptake, Discussion on $\mathrm{CO}_{2}$ eq emissions of an airplane calculations. Video SI1: Video showing the iPerf testing of Ethernet speeds of CNT Ethernet cable.

Author Contributions: Conceptualization, A.O.W.; methodology, AO.W.; software, A.A. and L.P.; validation, V.S.G, R.E.P., R.C. and M.L.D.; formal analysis, A.O.W.; investigation, A.H., T.Y., Y.N., S.L., V.S.G., L.P., G.S., A.C., J.S.C.; resources, R.E.P., M.L.D, A.O.W.; data curation, A.O.W.; writing-original draft preparation, A.O.W. and A.H.; writing-review and editing, A.O.W., R.E.P., M.L.D., R.C.,A.H., T.Y., Y.N., S.L., V.S.G., L.P., G.S., I.T.; visualization, A.O.W.; supervision, A.O.W., R.E.P., M.L.D., S.J.C.; project administration, A.O.W.; funding acquisition, A.O.W. All authors have read and agreed to the published version of the manuscript.

Funding: A.O.W. is funded through Sêr Cymru II Fellowship by the Welsh Government and the European Regional Development Fund (ERDF). A.O.W. and T.Y. acknowledges funding from Welsh Government Circular Economy Capital Fund FY 2020-21. A.H. was funded by the Copper Nanotube Ultraconductive (UCC) wire project funded by Sêr Cymru National Research Network for Advanced Engineering and Materials (NRN) with contributions from E-Corp. G.S. received funding from Swansea Employability Academy (SEA) via the summer placements scheme. Thanks to funding by Welsh Government for Knowledge Economy Skills Scholarships (KESS2), part funded by the Welsh Government's European Social Fund (ESF) convergence programme for West Wales and the Valleys. L.P. and I.T. funded through KESS2 and TRIMTABS Ltd. J.S.C. funded through KESS2 and Salts Healthcare Ltd. M.L.D and R.C. are grateful for the financial support of the EPSRC (EP/S001336/1) and for funding LCA software and database licenses. We would like to acknowledge the Life Cycle Analysis for Circular Economy (LCA4CE) Lab. J.S.C. \& V.S.G. funded thanks to Salts Healthcare Ltd. A.A. funded in part by the Swansea University Texas Strategic Partnership. The authors acknowledge access to the TEM provided by the Swansea University AIM Facility, funded in part by the EPSRC (EP/M028267/1), the European Regional Development Fund through the Welsh Government (80708) and the Welsh Government's Sêr Cymru program. The funders had no role in the design of the study; in the collection, analyses, or interpretation of data; in the writing of the manuscript, or in the decision to publish the results.

Acknowledgments: We would like to thank Keysight Technologies for the use of a test model of the B2900A SMU. We would like to acknowledge the assistance provided by Swansea University College of Engineering AIM Facility, which was funded in part by the EPSRC (EP/M028267/1), the European Regional Development Fund through the Welsh Government (80708) and the Sêr Solar project via Welsh Government. We would like to thank TRIMTABS Ltd for purchasing equipment required for making Ethernet cables. Thanks to Swansea Employability Academy (SEA) for the summer placements scheme. Thanks to the Swansea University Texas Strategic Partnership. R.E.P. acknowledges his work was associated with the IMPACT operation.

Conflicts of Interest: A.O.W. founded TRIMTABS Ltd who co-sponsored I.T. and L.P. through the KESS2 program. All other authors declare no conflict of interest. The funders had no role in the design of the study; in the collection, analyses, or interpretation of data; in the writing of the manuscript, or in the decision to publish the results.

\section{References}

1. Smalley, R.E. Future Global Energy Prosperity: The Terawatt Challenge. MRS Bull. 2005, 67, 412-417, doi:10.1557/mrs2005.124.

2. Anuar Sharuddin, S.D.; Abnisa, F.; Wan Daud, W.M.A.; Aroua, M.K. A Review on Pyrolysis of Plastic Wastes. Energy Convers. Manag. 2016, 115, 308-326, doi:10.1016/J.ENCONMAN.2016.02.037. 
3. Khodabakhshi, S.; Kiani, S.; Niu, Y.; White, A.O.; Suwaileh, W.; Palmer, R.E.; Barron, A.R.; Andreoli, E. Facile and Environmentally Friendly Synthesis of Ultramicroporous Carbon Spheres: A Significant Improvement in CVD Method. Carbon. 2021, 171, 426-436, doi:10.1016/j.carbon.2020.08.056.

4. Geyer, R.; Jambeck, J.R.; Law, K.L. Production, Uses, and Fate of All Plastics Ever Made. Sci. Adv. 2017, 3, 5, doi:10.1126/sciadv.1700782.

5. Bazargan, A.; McKay, G. A Review - Synthesis of Carbon Nanotubes from Plastic Wastes. Chem. Eng. J. 2012, 195-196, 377-391, doi:10.1016/j.cej.2012.03.077.

6. Acomb, J.C.; Wu, C.; Williams, P.T. Control of Steam Input to the Pyrolysis-Gasification of Waste Plastics for Improved Production of Hydrogen or Carbon Nanotubes. Appl. Catal. B Environ. 2014, 147, 571-584, doi:10.1016/J.APCATB.2013.09.018.

7. Aboul-Enein, A.A.; Adel-Rahman, H.; Haggar, A.M.; Awadallah, A.E. Simple Method for Synthesis of Carbon Nanotubes over Ni-Mo/Al 2 O 3 Catalyst via Pyrolysis of Polyethylene Waste Using a Two-Stage Process. Fullerenes, Nanotub. Carbon Nanostructures 2017, 25, 211-222, doi:10.1080/1536383X.2016.1277422.

8. El Essawy, N.A.; Konsowa, A.H.; Elnouby, M.; Farag, H.A. A Novel One-Step Synthesis for Carbon-Based Nanomaterials from Polyethylene Terephthalate (PET) Bottles Waste. J. Air Waste Manage. Assoc. 2017, 67, 358-370, doi:10.1080/10962247.2016.1242517.

9. Liu, X.; Sun, H.; Wu, C.; Patel, D.; Huang, J. Thermal Chemical Conversion of High-Density Polyethylene for the Production of Valuable Carbon Nanotubes Using Ni/AAO Membrane Catalyst. Energy and Fuels 2018, 32, 4511-4520, doi:10.1021/acs.energyfuels.7b03160.

10. Arena, U.; Mastellone, M.L.; Camino, G.; Boccaleri, E. An Innovative Process for Mass Production of Multi-Wall Carbon Nanotubes by Means of Low-Cost Pyrolysis of Polyolefins. Polym. Degrad. Stab. 2006, 91, 763-768, doi:10.1016/j.polymdegradstab.2005.05.029.

11. Miller-Chou, B.A.; Koenig, J.L. A Review of Polymer Dissolution. Prog. Polym. Sci. 2003, 28, 1223-1270.

12. Puengjinda, P.; Sano, N.; Tanthapanichakoon, W.; Charinpanitkul, T. Selective Synthesis of Carbon Nanotubes and Nanocapsules Using Naphthalene Pyrolysis Assisted with Ferrocene. J. Ind. Eng. Chem. 2009, 15, 375-380, doi:10.1016/j.jiec.2008.11.003.

13. Charinpanitkul, T.; Sano, N.; Puengjinda, P.; Klanwan, J.; Akrapattangkul, N.; Tanthapanichakoon, W. Naphthalene as an Alternative Carbon Source for Pyrolytic Synthesis of Carbon Nanostructures. J. Anal. Appl. Pyrolysis 2009, 86, 386-390, doi:10.1016/j.jaap.2009.08.001.

14. Orbaek, A.W.; Barron, A.R. Towards a 'Catalyst Activity Map' Regarding the Nucleation and Growth of Single Walled Carbon Nanotubes. J. Exp. Nanosci. 2015, 10, 66-76, doi:10.1080/17458080.2013.794979.

15. Hedayati, A.; Barnett, C.; Swan, G.; Orbaek White, A. Chemical Recycling of Consumer-Grade Black Plastic into Electrically Conductive Carbon Nanotubes. C 2019, 5, 32, doi:10.3390/c5020032.

16. Narasimhan, B.; Peppas, N.A. Disentanglement and Reptation during Dissolution of Rubbery Polymers. J. Polym. Sci. Part B Polym. Phys. 1996, 34, 947-961, doi:10.1002/(SICI)1099-0488(19960415)34:5<947::AID-POLB13>3.0.CO;2-B.

17. Achilias, D.S.; Giannoulis, A.; Papageorgiou, G.Z. Recycling of Polymers from Plastic Packaging Materials Using the Dissolution-Reprecipitation Technique. Polym. Bull. 2009, 63, 449-465, doi:10.1007/s00289-009-0104-5.

18. Bjørn A., Owsianiak M., Molin C., Laurent A. Main Characteristics of LCA. In Life Cycle Assessment Theory and Practice, 1st ed., Hauschild M., Rosenbaum R., Olsen S. (eds), Springer, cham, 2018, pp.9-16.

19. Heidari, A.; Younesi, H. Synthesis, Characterization and Life Cycle Assessment of Carbon Nanospheres from Waste Tires Pyrolysis over Ferrocene Catalyst. J. Environ. Chem. Eng. 2020, 8, 103669, doi:10.1016/j.jece.2020.103669.

20. Rybicka, J.; Tiwari, A.; Leeke, G.A. Technology Readiness Level Assessment of Composites Recycling Technologies. J. Clean. Prod. 2016, 112, 1001-1012, doi:10.1016/j.jclepro.2015.08.104.

21. Al-Salem, S.M.; Lettieri, P.; Baeyens, J. Recycling and Recovery Routes of Plastic Solid Waste (PSW): A Review. Waste Manag. 2009, 29, 2625-2643, doi:10.1016/j.wasman.2009.06.004.

22. Kwon, J.-H.; Kim, J.-W.; Pham, T.D.; Tarafdar, A.; Hong, S.; Chun, S.-H.; Lee, S.-H.; Kang, D.-Y.; Kim, J.-Y.; Kim, S.-B.; et al. Microplastics in Food: A Review on Analytical Methods and Challenges. Int. J. Environ. Res. Public Health 2020, 17, 1-23, doi:10.3390/IJERPH17186710.

23. Orbaek, A.W.; Aggarwal, N.; Barron, A.R. The Development of a 'Process Map' for the Growth of Carbon Nanomaterials from Ferrocene by Injection CVD. J. Mater. Chem. A 2013, 1, 14122, doi:10.1039/c3ta13543h.

24. Gangoli, V.S.; Raja, P.M. V.; Esquenazi, G.L.; Barron, A.R. The Safe Handling of Bulk Low-Density Nanomaterials. SN Appl. Sci. 2019, 1, 644, doi:10.1007/s42452-019-0647-5.

25. Dee, N.T.; Li, J.; White, A.O.; Jacob, C.; Shi, W.; Kidambi, P.R.; Cui, K.; Zakharov, D.N.; Janković, N.Z.; Bedewy, M.; et al. CarbonAssisted Catalyst Pretreatment Enables Straightforward Synthesis of High-Density Carbon Nanotube Forests. Carbon N. Y. 2019, doi:10.1016/j.carbon.2019.06.083.

26. Rueden, C.T.; Schindelin, J.; Hiner, M.C.; DeZonia, B.E.; Walter, A.E.; Arena, E.T.; Eliceiri, K.W. ImageJ2: ImageJ for the next Generation of Scientific Image Data. BMC Bioinforma. 2017, 18, 18, 1-26, doi:10.1186/S12859-017-1934-Z.

27. King, S.G.; McCafferty, L.; Stolojan, V.; Silva, S.R.P. Highly Aligned Arrays of Super Resilient Carbon Nanotubes by Steam Purification. Carbon N. Y. 2015, 84, 130-137, doi:10.1016/j.carbon.2014.11.061.

28. Chiang, I.W.; Brinson, B.E.; Smalley, R.E.; Margrave, J.L.; Hauge, R.H. Purification and Characterization of Single-Wall Carbon Nanotubes. J. Phys. Chem. B 2001, 105, 1157-1161, doi:10.1021/jp003453z.

29. Mansfield, E.; Kar, A.; Hooker, S.A. Applications of TGA in Quality Control of SWCNTs. Anal. Bioanal. Chem. 2010, 396, 10711077, doi:10.1007/s00216-009-3319-2.

30. Khodabakhhshi, S.; Fulvio, P.F.; Sousaraei, A.; Kiani, S.; Niu, Y.; Palmer, R.E.; Kuo, W.C.H.; Rudd, J.; Barron, A.R.; Andreoli, E. Oxidative Synthesis of Yellow Photoluminescent Carbon Nanoribbons from Carbon Black. Carbon N. Y. 2021, 183, 495-503, doi:10.1016/J.CARBON.2021.07.032. 
31. Andreoli, E.; Suzuki, R.; Orbaek, A.W.; Bhutani, M.S.; Hauge, R.H.; Adams, W.; Fleming, J.B.; Barron, A.R. Preparation and Evaluation of Polyethyleneimine-Single Walled Carbon Nanotube Conjugates as Vectors for Pancreatic Cancer Treatment. J. Mater. Chem. B 2014, 2, 4740, doi:10.1039/c4tb00778f.

32. Chiang, I.W.; Brinson, B.E.; Huang, A.Y.; Willis, P.A.; Bronikowski, M.J.; Margrave, J.L.; Smalley, R.E.; Hauge, R.H. Purification and Characterization of Single-Wall Carbon Nanotubes (SWNTs) Obtained from the Gas-Phase Decomposition of CO (HiPco Process). J. Phys. Chem. B 2001, 105, 8297-8301, doi:10.1021/jp0114891.

33. López-Lorente, A.I.; Simonet, B.M.; Valcárcel, M. The Potential of Carbon Nanotube Membranes for Analytical Separations. Anal. Chem. 2010, 82, 5399-5407, doi:10.1021/ac902629n.

34. Aircraft Electrical Wire, Available online: aircraft_electrical_wire.pdf (mitrecaasd.org) (accessed on 5th May 2021)

35. Lu, Q.; Keskar, G.; Ciocan, R.; Rao, R.; Mathur, R.B.; Rao, A.M.; Larcom, L.L. Determination of Carbon Nanotube Density by Gradient Sedimentation. J. Phys. Chem. B 2006, 110, 24371-24376, doi:10.1021/jp063660k.

36. Dresselhaus, M.S.; Dresselhaus, G.; Jorio, A. Raman Spectroscopy of Carbon Nanotubes in 1997 and 2007. J. Phys. Chem. C 2007, 111, 17887-17893, doi:10.1021/jp071378n

37. Lu, Q.; Keskar, G.; Ciocan, R.; Rao, R.; Mathur, R.B.; Rao, A.M.; Larcom, L.L. Determination of Carbon Nanotube Density by Gradient Sedimentation. J. Phys. Chem. B 2006, 110, 24371-24376, doi:10.1021/jp063660k.

38. Box Plot. https://www.itl.nist.gov/div898/handbook/eda/section3/boxplot.htm (Accessed on 21 October, 2021)

39. Meam Plot. https://www.itl.nist.gov/div898/handbook/eda/section3/meanplot.htm (Accessed on 21 Oct, 2022)

40. Baker, R.T.K. Catalytic Growth of Carbon Filaments. Carbon N. Y. 1989, 27, 315-323, doi:10.1016/0008-6223(89)90062-6.

41. Wood, R.F.; Pannala, S.; Wells, J.C.; Puretzky, a. a.; Geohegan, D.B. Simple Model of the Interrelation between Single- and Multiwall Carbon Nanotube Growth Rates for the CVD Process. Phys. Rev. B 2007, 75, 235446, doi:10.1103/PhysRevB.75.235446.

42. Somorjai, G.A.; Carrazza, J. Structure Sensitivity of Catalytic Reactions. Ind. Eng. Chem. Fundam. 1986, 25, 63-69, doi:10.1021/i100021a009.

43. Rao, R.; Liptak, D.; Cherukuri, T.; Yakobson, B.I.; Maruyama, B. In Situ Evidence for Chirality-Dependent Growth Rates of Individual Carbon Nanotubes. Nat. Mater. 2012, 11, 213-216, doi:10.1038/nmat3231.

44. Marchand, M.; Journet, C.; Guillot, D.; Benoit, J.-M.; Yakobson, B.I.; Purcell, S.T. Growing a Carbon Nanotube Atom by Atom: “And Yet It Does Turn." Nano Lett. 2009, 9, 2961-2966, doi:10.1021/n1901380u.

45. Matsugi, A. Thermal Decomposition of Benzyl Radicals: Kinetics and Spectroscopy in a Shock Tube. J. Phys. Chem. A 2020, 124, 824-835, doi:10.1021/acs.jpca.9b10705.

46. Brouwer, L.D.; Mueller-Markgraf, W.; Troe, J. Thermal Decomposition of Toluene: A Comparison of Thermal and Laser-Photochemical Activation Experiments. J. Phys. Chem. 1988, 92, 4905-4914, doi:10.1021/j100328a021.

47. Oehlschlaeger, M.A.; Davidson, D.F.; Hanson, R.K. Thermal Decomposition of Toluene: Overall Rate and Branching Ratio. Proc. Combust. Inst. 2007, 31, 211-219, doi:10.1016/J.PROCI.2006.07.002.

48. Maafa,I.M. Pyrolysisof Polystyrene Waste: A Review. Polymers 2021, 13, 225. https:// doi.org/10.3390/polym13020225

49. Fürstner, A. Iron Catalysis in Organic Synthesis: A Critical Assessment of What It Takes To Make This Base Metal a Multitasking Champion. ACS Cent. Sci. 2016, 2, 778-789, doi:10.1021/acscentsci.6b00272.

50. Williams, P.T. Hydrogen and Carbon Nanotubes from Pyrolysis-Catalysis of Waste Plastics: A Review. Waste and Biomass Valorization 2021, 12, 1-28, doi:10.1007/s12649-020-01054-w.

51. Barnett, C.J.; McCormack, J.E.; Deemer, E.M.; Evans, C.R.; Evans, J.E.; White, A.O.; Dunstan, P.R.; Chianelli, R.R.; Cobley, R.J.; Barron, A.R. Enhancement of Multiwalled Carbon Nanotubes' Electrical Conductivity Using Metal Nanoscale Copper Contacts and Its Implications for Carbon Nanotube-Enhanced Copper Conductivity. J. Phys. Chem. C 2020, 124, 18777-18783, doi:10.1021/acs.jpcc.0c05000.

52. Jarosz, P.; Schauerman, C.; Alvarenga, J.; Moses, B.; Mastrangelo, T.; Raffaelle, R.; Ridgley, R.; Landi, B. Carbon Nanotube Wires and Cables: Near-Term Applications and Future Perspectives. Nanoscale 2011, 3, 4542, doi:10.1039/c1nr10814j.

53. House of Commons Library, Broadband (https://commonslibrary.parliament.uk/broadband-faqs/) (accessed on Oct 27, 2021)

54. Upadhyayula, V.K.K.; Meyer, D.E.; Curran, M.A.; Gonzalez, M.A. Life Cycle Assessment as a Tool to Enhance the Environmental Performance of Carbon Nanotube Products: A Review. J. Clean. Prod. 2012, 26, 37-47, doi:10.1016/J.JCLEPRO.2011.12.018.

55. Griffiths, O.G.; O’Byrne, J.P.; Torrente-Murciano, L.; Jones, M.D.; Mattia, D.; McManus, M.C. Identifying the Largest Environmental Life Cycle Impacts during Carbon Nanotube Synthesis via Chemical Vapour Deposition. J. Clean. Prod. 2013, 42, 180189, doi:10.1016/J.JCLEPRO.2012.10.040.

56. Nagy, B.; Farmer, J.D.; Bui, Q.M.; Trancik, J.E. Statistical Basis for Predicting Technological Progress. PLoS One 2013, 8, e52669, doi:10.1371/journal.pone.0052669.

57. Pawelke, R.H. Marrying Wright's Law to Thermodynamics for an Ideal Relative Final Cost-Predicting Model of Carbon-Fuel Substitution. 2021, doi:10.26434/chemrxiv.12613049.v2.

58. Gavankar, S.; Suh, S.; Keller, A.A. The Role of Scale and Technology Maturity in Life Cycle Assessment of Emerging Technologies: A Case Study on Carbon Nanotubes. J. Ind. Ecol. 2015, 19, 51-60, doi:10.1111/jiec.12175.

59. Healy, M.L.; Dahlben, L.J.; Isaacs, J.A. Environmental Assessment of Single-Walled Carbon Nanotube Processes. J. Ind. Ecol. 2008, 12, 376-393, doi:10.1111/J.1530-9290.2008.00058.X. 